\title{
MOVILIDAD COTIDIANA Y ACCESO A LOS SERVICIOS CULTURALES, ESTUDIO DE CASO EN TECÁMAC, ESTADO DE MÉXICO ${ }^{1}$
}

DAILY MOBILITY AND ACCESS TO CULTURAL SERVICES. CASE STUDY IN TECÁMAC, STATE OF MEXICO.

\author{
Cuauhtémoc Ochoa ${ }^{2}$ \\ Georgina Isunza ${ }^{3}$ \\ Marco Antonio Morán ${ }^{4}$
}

\section{Resumen}

Se explora en este trabajo, la relación que existe entre las prácticas de movilidad cotidiana, la construcción de espacios de vida y el acceso a la cultura de los nuevos residentes que arriban a los espacios peri-urbanos de la Ciudad de México. Se realiza un estudio de caso en el municipio de Tecámac, localizado en el área periférica de la Ciudad de México donde ha tenido lugar el desarrollo de grandes complejos habitacionales que imponen severas dificultades a la movilidad cotidiana de sus habitantes.

El estudio utiliza recursos de la investigación cualitativa (entrevistas y relatos), así como de la aplicación de una encuesta que proporciona información sobre los hábitos de movilidad cotidiana de las personas y sus patrones de consumo cultural.

Palabras clave: movilidad cotidiana, espacios de vida, cultura, ciudad de México

\section{Abstract}

This paper explores the relationship between daily mobility practices, living spaces and access to the culture of new residents arriving in the peri-urban areas of Mexico City. A case study is carried out in the municipality of Tecámac, located in the peripheral area of the City of Mexico where there has been the development of large housing complexes that impose severe difficulties in daily mobility of their inhabitants.

The study uses qualitative research resources (interviews and stories) and the implementation of a survey that provides information on daily mobility habits of people and their cultural consumption patterns.

Keywords: daily mobility, living spaces, culture, Mexico City

\footnotetext{
${ }^{1}$ Se exponen aquí parte de los resultados del Proyecto SIP 20130405 "Desarrollos Inmobiliarios y movilidad residencial en la ZMVM, financiado por el Instituto Politécnico Nacional. Esta investigación se orientó a los conjuntos habitacionales localizados en la periferia urbana de la Zona Metropolitana del Valle de México (ZMVM).

2 Doctor en Ciencias Políticas y Sociales por la Universidad Nacional Autónoma de México. Profesor investigador en la Universidad Autónoma de la Ciudad de México (México).E-mail: cuauochoa@yahoo.com.mx

${ }^{3}$ Dra. En planificación territorial y desarrollo regional. Profesora e investigadora de la mestria en economía y gestión municipal. Subdirección académica y de investigación. E-mail: ginaisunza@yahoo.com.mx

${ }^{4}$ Maestro en Economía y Gestión Municipal. E-mail: moranvsx@gmail.com
} 


\section{INTRODUCCION}

En las metrópolis del siglo XXI, los estudios sobre la movilidad han cobrado creciente importancia y han transitado de la visión determinista del transporte, el viaje y la accesibilidad a las infraestructuras, para colocarse en el campo de las ciencias sociales como una transformación de la experiencia del habitar urbano. Ello ofrece un terreno fértil para la generación de nuevo conocimiento y la emergencia de temáticas que implican un dialogo entre distintas disciplinas. En especial, este trabajo se plantea contribuir a la discusión de la relación entre la movilidad, los espacios de vida y la cultura, a partir de un estudio de caso en Tecámac, municipio periurbano de la Zona Metropolitana del Valle de México $(Z M V M)^{5}$ que enfrenta graves problemas de movilidad cotidiana.

El objetivo de este trabajo es explorar la relación que existe entre las prácticas de movilidad cotidiana, la construcción de espacios de vida y el acceso a la cultura de los nuevos residentes que arriban a los espacios peri-urbanos de la Ciudad de México.

La aplicación de la encuesta "Movilidad Residencial y tendencias electorales en Tecámac y Zumpango, Estado de México" así como el empleo de los recursos metodológicos de la investigación cualitativa (entrevistas semi-estructuradas, relatos, observación participante) permiten tratar el tema de la movilidad y el acceso a la cultura con tal profundidad que fue posible identificar las prácticas habituales de movilidad y las pautas culturales de los nuevos residentes que arriban al municipio motivados por el acceso a una vivienda social.

El análisis del perfil de los nuevos residentes en Tecámac y sus patrones de movilidad cotidiana inspiraron una hipótesis que orienta la presente investigación: la movilidad por motivos residenciales no representa cambio en la demanda de bienes y servicios culturales. Por su origen socioeconómico y las pautas de consumo cultural previa, los nuevos residentes tienden a adaptarse a la oferta del entorno local de destino (Tecámac) que provee una oferta cultural similar a la que prevalecía en sus lugares de origen ya que muy pocos provienen de áreas de la metrópoli en las que se localiza una oferta cultural más diversa, amplia, al mismo tiempo especializada. Casi toda la oferta cultural que podríamos llamar "clásica" (centros educativos, librerías, museos, salas de teatro y cineclubes) se halla concentrada en la ciudad central (ver Mapa 1), especialmente en el triángulo que forman el área del parque de Chapultepec en el poniente, el Zócalo en el centro de la ciudad y la zona

\footnotetext{
${ }^{5}$ La Zona Metropolitana del Valle de México es un conglomerado territorial que encabeza el sistema urbano en México. De acuerdo con el Consejo Nacional de Población (CONAPO) La ZMVM comprende las 16 delegaciones del Distrito Federal (capital mexicana), 59 municipios del Estado de México y un municipio del Estado de Hidalgo (Tizayuca).
} 
de la Ciudad Universitaria en el sur; esta última zona ha incrementado su importancia de forma vertiginosa tanto en cantidad como en calidad.

La combinación de las diferencias de ingreso y nivel escolar aunado al irregular y complejo desarrollo urbano genera procesos de segregación cultural y de escaso aprovechamiento de muchos de los servicios existentes. Este desajuste entre la distribución poblacional y la distribución del equipamiento cultural crea, además, condiciones muy desiguales de desarrollo entre bienes simbólicos situados, que requieren la asistencia al lugar (desde universidades hasta cines y salones de bailes) y los medios de comunicación electrónica que llevan los bienes simbólicos a domicilio (radio, televisión, etc. $)^{6}$, por lo tanto, la cultura no está exenta del proceso de segregación que experimenta una metrópoli diversa y desigual.

Este proceso de adaptación se explica por la asimilación de políticas culturales dominantes instaladas en la infraestructura cultural de la ZMVM, que se tienden a reproducir en los espacios locales donde se hace evidente la divergencia entre la cultura institucional y la cultura de los ciudadanos. Salta a la vista el poco interés que dentro de las agendas locales se le concede a la cultura, además de la escasa capacidad de gestión del gobierno municipal para diversificar la oferta cultural.

En las últimas décadas, no obstante los esfuerzos de autoridades gubernamentales, agentes privados y organizaciones de la sociedad civil, se mantiene una baja correspondencia entre el crecimiento urbano y la estructura y distribución de los equipamientos culturales. Casi toda la oferta cultural que podríamos Ilamar "clásica" (centros educativos, librerías, museos, salas de teatro y cineclubes) se halla concentrada en el triángulo que forman el área del parque de Chapultepec en el poniente, el Zócalo en el centro de la ciudad y la zona de la Ciudad Universitaria en el sur; esta última zona ha incrementado su importancia de forma vertiginosa tanto en cantidad como en calidad. La combinación de las diferencias de ingreso y nivel escolar aunado al irregular y complejo desarrollo urbano genera procesos de segregación cultural y de escaso aprovechamiento de muchos de los servicios existentes. Este desajuste entre el mapa de distribución poblacional y el de distribución de equipamiento cultural crea, además, condiciones muy desiguales de desarrollo entre bienes simbólicos situados, que requieren la asistencia al lugar (desde universidades hasta cines y salones de bailes) y los medios de comunicación electrónica que llevan los bienes simbólicos a domicilio (radio, televisión, etc.).

\footnotetext{
${ }^{6}$ Véanse los trabajos de García Canclini (1993); Preciat, (1997) y Conaculta (2015).
} 


\section{MOVILIDAD Y CULTURA URBANA}

Cuando Louis Wirth (1938) $)^{7}$ analiza el dominio de la gran ciudad, se refiere a una alta concentración de servicios y actividades industriales, comerciales, financieras y administrativas; de líneas de transporte y comunicación; de equipamiento y servicios culturales y recreativos tales como la prensa, estaciones de radio, teatros, bibliotecas, museos, salas de conciertos, teatros líricos, hospitales, instituciones de educación superior, centros de investigaciones, publicidad, organizaciones profesionales e instituciones religiosas y de beneficencia. Por lo tanto, la ciudad ejerce atracción sobre la población rural y la incorporada a su sistema de vida. Los nuevos pobladores de la ciudad, quizá portadores inicialmente, de elementos tradicionales, terminan asimilando el estilo de vida urbano, o bien, crean otras formas de habitar que configuran un mosaico heterogéneo y multiculural. Pero qué sucede cuando los ciclos urbanos superan la tendencia principal de migración rural-urbana dando cabida a una compleja red de movimientos temporales y permanentes donde predomina la migración urbana-urbana, entre ciudades de distinto rango hacia las metrópolis y al interior de las metrópolis, de zonas de alta centralidad hacia espacios periurbanos cada vez más lejanos? Ello plantea varias interrogantes, bajo el entendido de que una ciudad de escala metropolitana es tan compleja que da lugar a diversas formas de habitar y de organizar socialmente el espacio. La complejidad de los flujos y las interacciones culturales han dado lugar a los estudios de movilidad urbana desde la perspectiva de las prácticas sociales cotidianas.

Entender el efecto que tiene la movilidad sobre los modos de vida de los habitantes urbanos, inspira nuevas formas de entender "lo urbano" desde una perspectiva sociocultural, es decir, la movilidad es un componente fundamental en la conformación de una cultura propiamente urbana (Lange, 2011, p.91).

Evocando a los sociólogos de Chicago, Carlos Lange señala que la cultura urbana implica: Nuevas formas de sociabilidad caracterizadas por el predominio de vínculos sociales precarios, fragmentarios, transitorios, dispersos y con fuerte énfasis del anonimato, los cuales influyen en la conformación de normas, costumbres, tradiciones e instituciones diferenciadas y heterogéneas, de transformación constante y cada vez más acelerada a lo largo del tiempo (Lange, 2011, p.92).

Entre las distintas formas de movilidad, los desplazamientos cotidianos constituyen importantes factores de la organización y de la construcción de territorios, por lo que el estudio de movilidad espacial permite profundizar el conocimiento de las transformaciones territoriales que cobran mayor relevancia en el presente siglo, especialmente en las metrópolis. De acuerdo con

\footnotetext{
7 Sociólogo considerado uno de los principales exponentes de la Escuela de Chicago, autor de "El urbanismo como modo de vida" publicado en American Journal of Sociology n44, en 1938.
} 
Casado (2008) la movilidad cotidiana se identifica con las prácticas habituales y reiteradas de desplazamientos de corta duración y distancia vinculadas a distintos fines.

La construcción de territorio tiene entonces una estrecha relación con los espacios de vida, en tanto lugares concretos donde tienen lugar movimientos cotidianos motivados por distintas necesidades (Susino, 2001). Predominan los desplazamientos al trabajo y al lugar de estudio, las compras, visitas a familiares y amigos, traslado de los niños al colegio, gestiones administrativas (trámites), el acceso a servicios médicos y el ocio, que implica el acceso a los servicios culturales, entre otras.

Una ciudad metropolitana constituye un espacio de vida colectivo y un espacio de probabilidades de ocurrencia de determinados desplazamientos (Susino, 2001). La percepción de los espacios de vida es una valoración subjetiva de los actores sociales y la indeterminación de sus límites de tal manera que la inserción social de un individuo dependerá en buena medida, de la capacidad y las posibilidades que tenga éste para desplazarse; por lo tanto, la construcción de los espacios de vida supone el acceso a la ciudad, en el entendido de que las prácticas de movilidad incluyen, excluyen, condicionan, habilitan, inhiben o potencian el uso de la ciudad" (Blanco, Bosoer y Apaolaza, 2014, p.2).

Los desplazamientos cotidianos permiten a las personas entrar en contacto con distintos ambientes que sólo conocen fragmentariamente y en los que interaccionan con otros individuos de forma parcial. Esta situación propicia cierto aislamiento de los individuos en su hogar, reforzando el protagonismo de la familia y las estrategias particulares frente a los mecanismos de solidaridad vecinal y colectiva (Lange, 2011, p.92).

La movilidad también se relaciona con la habitabilidad. Un espacio habitable permite que la vida cotidiana se organice dentro de un espacio de vida polifuncional próximo al hogar, e involucra el ambiente sociocultural: prácticas sociales cotidianas, pautas de consumo, la identidad, los lazos sociales y el imaginario colectivo. Así, los espacios construidos deben tener la capacidad para satisfacer las necesidades objetivas y subjetivas de los individuos y grupos sociales. El entorno social y ambiental debería contribuir a percibir bienestar personal y colectivo por residir en un barrio determinado (Zulaica y Celemín, 1988, p.130-131)

La divergencia entre la cultura institucional y la cultura de los ciudadanos impide la polifuncionalidad del espacio e implica justamente la disociación entre las acciones institucionales y las características económicas y sociales de las comunidades locales. La separación de las funciones y de los sujetos, así como la separación entre los sujetos mismos, suponen una enajenación de los ciudadanos en el diseño y uso del espacio, servicios y actividades. Asimismo, supone la exclusión de 
determinadas condiciones sociales emergentes, que impiden la construcción de la identidad y del territorio (Alguacil, 2001, p.6).

Así, la movilidad cotidiana no se limita sólo a los desplazamientos, también se relaciona con las facilidades o dificultades que enfrentan las personas para hacer posible sus desplazamientos cotidianos. Para este trabajo, es relevante el papel de los gobiernos locales para atender la demanda creciente de movilidad de los nuevos habitantes "[...] que se disputan la prioridad entre el automóvil privado y el servicio de transporte público" (Avellaneda y Lazo, 2011 p. 49).

Ante un patrón de urbanización disperso, la fragmentación urbana implica la fragmentación del tiempo social. En efecto, la fragmentación urbana ${ }^{8}$ característica de las transformaciones de las grandes metrópolis en el mundo, al amparo de políticas de desregulación del crecimiento urbano, financiarización y protagonismo del gran capital inmobiliario, han favorecido la construcción de vivienda en núcleos aislados desprovistos de los beneficios de la ciudad. Ello ha producido severos efectos en el uso del tiempo que las personas emplean para desplazarse, agravado por la falta de accesibilidad a las infraestructuras del transporte.

Así, la fragmentación del tiempo y del espacio se expresan en la falta de integración sectorial; la concentración y jerarquización del poder que desde la globalización económica supone el distanciamiento y aislamiento de los sujetos frente a los procesos económicos y sociales en los que se inscriben. La racionalidad separada desde la homogeneización de la cultura, condena a la enajenación del sujeto del control de los procesos sociales e incide en la desvertebración de los tejidos sociales (Alguacil, 2001:6).

El uso social del territorio influye en el tiempo cotidiano, ya que los lugares donde se trabaja, se compra, se vive, dibujan un conjunto de interrelaciones y de conexiones. En términos sociológicos, se trata de interpretar el territorio metropolitano incluyendo los destinos de las actividades que dibujan la cotidianidad en su conjunto y no sólo el trabajo (Miralles, 2011, p.130)

En consecuencia, el tiempo de la movilidad se valora desde una perspectiva global de los tiempos y desde la realidad cotidiana de los ciudadanos, integrada en el conjunto de actividades que consumen tiempo. El tiempo del desplazamiento no es un valor cuantitativo y aislado porque se interpreta en relación a las otras esferas del tiempo. Desde la perspectiva del ciudadano, las diversas actividades y el tiempo que les dedican no están aislados: el tiempo cotidiano, el de los desplazamientos de la proximidad o de la lejanía, y del territorio contiguo o diseminado, es el tiempo

\footnotetext{
${ }^{8}$ La fragmentación urbana expresa la ruptura en el concepto integral de ciudad; segmentación y atomización del espacio urbano, discontinuidades, lógicas de separación y fronteras urbanas que se traducen en la diferenciación de grupos sociales. (Jirón y Mansilla, 2014).
} 
de la vida cotidiana, donde confluyen (en competencia o complementariedad) las distintas actividades (Miralles, 2011, p.129)

\section{PROGRAMAS HABITACIONALES Y MOVILIDAD RESIDENCIAL}

En México, los programas habitacionales han condicionado el acceso a la vivienda social en lugares que rompen con el espacio de vida original de las familias; en este sentido, los cambios en el lugar de residencia de las familias, se relacionan con los cambios sociodemográficos y con las formas de acceso a la vivienda; al respecto Módenes (2004, p.9) asegura:

La movilidad residencial es la consecuencia espacial de la gestión residencial que efectúan los hogares para poner de acuerdo las distintas esferas de su vida cotidiana: la función estrictamente residencial de la vivienda, la relación con el lugar de trabajo, las tareas de mantenimiento del hogar y de relaciones sociales, tanto por lo que respecta a la reproducción demográfica como el sostén y el uso de las redes familiares y de amistad.

La ciudad de México, al igual que numerosas metrópolis latinoamericanas, ha experimentado grandes desarrollos inmobiliarios desde la última década del siglo pasado. Este proceso ha favorecido el crecimiento urbano cada vez más fragmentado y disperso mediante el incremento de los flujos de población cuyo destino principal es la periferia rural-urbana de la ciudad de México, así como la incorporación de suelo antes dedicado a actividades agropecuarias de subsistencia, para el uso habitacional. La naturaleza, intensidad, dirección y área de influencia de estos flujos imprime sentido al análisis de la movilidad y los espacios de vida que en este caso, tienen alcance metropolitano.

Por otro lado, las normas y criterios que deben cumplir estos conjuntos localizados en la periferia urbana de la ciudad metrópoli, son definidos en el Código Administrativo del Estado de México (CAEM) en el cual se establecen con un grado de generalidad que implica, como apunta Duhau $(2008$, p. 21):

Que más allá de las especificaciones que en cada caso negocie el desarrollador con las autoridades competentes, las características de la estructura urbana resultante (traza urbana, estructura vial, infraestructura, distribución de usos del suelo, equipamientos, tamaño de los lotes) constituye una suerte de caja negra cuyo contenido ha quedado librado a la obligación genérica de que las empresas desarrolladoras deberán "construir obras de infraestructura, urbanización y equipamiento urbano conforme a [...] los proyectos, especificaciones y plazos autorizados por la Secretaría de Desarrollo Urbano y Obras Públicas y demás autoridades competentes" (Código Administrativo del Estado de México, p.21). 
Así, diversos aspectos importantes para la habitabilidad como los equipamientos sociales, culturales y de entretenimiento, no fueron o no serán considerados en su diseño, construcción y funcionamiento, pues a criterio implícito de los desarrolladores serán aspectos que los propios habitantes o autoridades municipales o estatales deberán de resolver según la demanda y necesidades de los propios habitantes. Es decir, las necesidades individuales serán resueltas en el marco del mercado y la intervención marginal del Estado. Es claro en este sentido que el modelo de regulación sólo establece requisitos elementales a las características que habrán de tener las viviendas. "En este modelo la provisión y el mejoramiento del funcionamiento de los servicios urbanos y los productos ofrecidos en la adquisición de la vivienda se darán a partir de la competencia entre los desarrolladores inmobiliarios (Duhau, 2008, p.25-26).

Los procesos urbanos de las últimas décadas han producido o acentuado los procesos de desarticulación y segregación socioespacial con la construcción, cada vez más frecuentes de espacios cerrados o aislados de su entorno, tanto en el interior de la ciudad como en sus zonas periféricas. Esta tendencia no sólo se observa en espacios construidos para que habiten sectores medios y altos sino también de sectores populares.

La búsqueda de suelo barato para desarrollar conjuntos de gran tamaño lleva a las empresas involucradas, a adquirir grandes predios en zonas cada vez más alejadas de las ciudades, lo cual deriva en la gestación de nuevos asentamientos, apartados de diversos servicios y equipamientos, y que a su vez se traduce en una dosis variable de exclusión para las familias alojadas (Boils, 2008, p. 282).

Esta exclusión se expresa con frecuencia en problemas de accesibilidad urbana, es decir, en nuestro estudio de caso, en el acceso limitado o nulo a los equipamientos culturales, a las instalaciones hospitalarias y de educación media superior o superior.

Por accesibilidad urbana, nos referimos al conjunto de atributos y de capacidades que garantizan a toda la población los beneficios de la vida urbana. Así, la accesibilidad se refiere no sólo a la capacidad de desplazamiento de las personas, "sino al conjunto de los dispositivos que promueven, permiten, estimulan y alientan al uso social del espacio urbano, de las infraestructuras y de los equipamientos" (Santos y Ganges y De Las Rivas, 2008, p. 21)

El modelo urbanístico en el que se inspiran los conjunto urbanos de vivienda de interés social característico del Municipio de Tecámac, se cataloga como un 'urbanismos insular' en la medida en que se orienta a producir estructuras urbanas sólo vinculadas a su entorno y al espacio metropolitano por medio de alguna vialidad colectora (Duhau, y Giglia, 2010),

Bajo las consideraciones expuestas, se plantea el problema en Tecámac, porque el entorno local no ofrece las condiciones de habitabilidad y el acceso a la cultura generalmente se limita a dos 
alternativas: las familias se adaptan a la escasa oferta cultural y recreativa que ofrecen los fraccionamientos; o bien, realizan desplazamientos a otros municipios o al Distrito Federal para realizar sus consumos culturales.

Las condiciones de habitabilidad y de movilidad, que ofrezcan los entornos locales, resultan claves para garantizar el derecho a la ciudad, por lo tanto, a los servicios culturales. En el caso de Tecámac, gran parte de la vida cotidiana no se organiza en el entorno local (espacio de proximidad) sino a lo largo y ancho del espacio metropolitano, por lo que "...en la ciudad contemporánea el espacio cotidiano se vuelve mucho más difuso y disperso" (Avellaneda y Lazo, 2011, p. 55).

Con el fin de tener una aproximación de los patrones de movilidad y el consumo cultural de los residentes de los grandes conjuntos habitacionales, localizados en la zona de estudio retomamos una parte de la Encuesta "Movilidad Residencial y tendencias electorales en Tecámac y Zumpango, Estado de México" (CIECAS, 2013), la cual revela qué tan dispuestas están las personas a desplazarse para realizar actividades lúdicas o recreativas. Otra fuente de información la constituyen los relatos de algunos residentes del municipio.

\section{PERFIL SOCIODEMOGRAFICO DE TECAMAC}

Tecámac es un municipio inserto en la dinámica metropolitana de la Ciudad de México y forma parte del tercer contorno metropolitano. Ha recibido importantes contingentes de población proveniente de otras áreas de la ciudad, como consecuencia de la proliferación de grandes desarrollos habitacionales (Mapa 1). 
Mapa 1. Localización del Municipio de Tecámac en la Zona Metropolitana del Valle de México

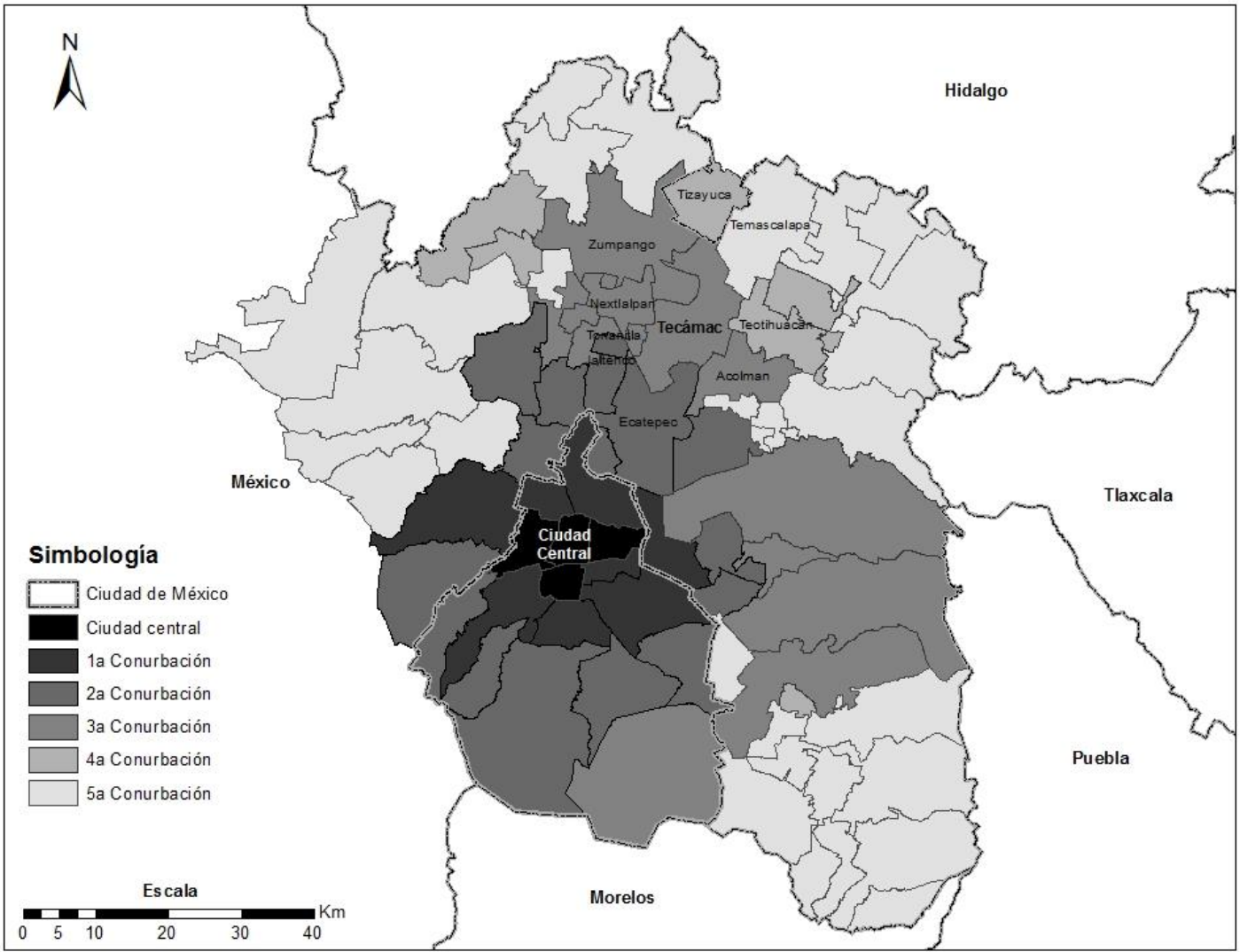

Elaboró: Mtro Maro A Morin

INEGI, Marco Geoes tadis tico Es tatal, 2010

Fuente: Elaboró Marco Morán V. con base en: INEGI, Marco Geoestadístico Estatal y Municipal 2010.

En el Cuadro 1 se pueden observar las tasas de crecimiento de Tecámac en relación con la tendencia de la ZMVM, del Distrito Federal (en donde se localizan las áreas de mayor centralidad) y los municipios metropolitanos. El Distrito Federal ha alcanzado una relativa estabilidad demográfica con tasas cercanas a cero. En contraste, destaca Tecámac, con una franca expansión demográfica en todos los periodos, especialmente desde el año 2000.

Cuadro 1. Tecámac y ZMVM, Tasa de crecimiento medio anual por periodo quinquenal 1990-2010.

\begin{tabular}{|l|c|c|c|c|}
\hline & Distrito Federal & Municipios conurbados & ZMVM & Tecámac \\
\hline $1990-1995$ & 0.6 & 3.0 & 1.9 & 3.8 \\
\hline $1995-2000$ & 0.3 & 2.0 & 1.2 & 3.1 \\
\hline $2000-2005$ & 0.3 & 1.4 & 0.9 & 9.4 \\
\hline $2005-2010$ & 0.3 & 1.4 & 0.9 & 6.1 \\
\hline
\end{tabular}

Fuente: Elaboración propia con base en INEGI, Censos de Población y Vivienda 1990, 2000 y 2010, y Conteos de Población y Vivienda 1995 y 2005. 
Como consecuencia del crecimiento demográfico el número de viviendas se incrementó 11.6\% en el periodo $2000-2005$ y $7.8 \%$ en el periodo 2005-2010; mientras que el número de hogares crecieron 10.1 y 9.2 en los periodos respectivos (INEGI, 2000, 2005 y 2010).

El comportamiento demográfico se relaciona con la intensa actividad inmobiliaria derivada de la construcción masiva de vivienda. En el periodo 2000-2010, los municipios del Estado de México pertenecientes a la ZMVM, atrajeron la construcción de 338 desarrollos habitacionales con 635 mil viviendas; Tecámac encabeza la lista con 42 de esos desarrollos, por lo que pasó de 38.8 mil viviendas registradas en el año censal 2000, a poco más de 97 mil en el 2010 (incremento mayor al 60\%). En consecuencia, la población de Tecámac registró un incremento absoluto cercano a 192 mil habitantes en el periodo intercensal (INEGI, 2010).

Por su parte, la actividad económica de Tecámac se ha trasformado significativamente, pasando de actividades de agricultura y ganadería a actividades del comercio en pequeña escala y servicios principalmente. Según el Censo Económico (INEGI, 2009), el sector primario sólo absorbía el $2 \%$ del empleo, el sector secundario el $36 \%$ y el terciario el $62 \%$.

Un breve diagnóstico de los hábitos de movilidad de la población del municipio a la luz de la Encuesta Origen Destino (INEGI, 2007) ${ }^{9}$ muestra que Tecámac daba origen a 384,448 viajes, teniendo como principales motivos el trabajo, regreso a casa y estudio, aunque cabe señalar que los viajes generados por motivo social o diversión ocupan el 5\% con más de 19 mil viajes (Cuadro 2)

Cuadro 2. Tecámac, viajes por propósito

\begin{tabular}{|c|c|c|}
\hline Propósito del viaje & $N^{\circ}$ de viajes & $\%$ \\
\hline Trabajo & 93,657 & 24.4 \\
\hline Regresar a casa & 165,751 & 43.1 \\
\hline Ir a estudiar & 33,185 & 8.6 \\
\hline Compras & 23,399 & 6.1 \\
\hline Llevar o recoger a alguien & 25,189 & 6.6 \\
\hline Social o diversión & 19,283 & 5.0 \\
\hline Relacionado con el trabajo & 1,961 & 0.5 \\
\hline Ir a comer & 1,449 & 0.4 \\
\hline Tramite & 4,965 & 1.3 \\
\hline Otros & 15,609 & 4.1 \\
\hline Total & 384,448 & 100 \\
\hline
\end{tabular}

Fuente: Tomado de Morán (2014:43)

\footnotetext{
${ }^{9}$ Contiene información sobre motivo del viaje, viajes producidos y atraídos, modo de transporte, tiempo, horario y características socio-demográficas de los usuarios de transporte. Su cobertura geográfica es la Zona Metropolitana del Valle de México de CONAPO-INEGI-SEDESOL (2004). Permite hacer análisis de flujos cotidianos, modelos de demanda de transporte y de articulación regional en torno al transporte.
} 
Los traslados originados en este municipio y que tenían como destino el Distrito Federal eran cerca de 62,749 , es decir un $16 \%$, mientras que 313,607 tenían como destino otros municipios del Estado de México (Morán, 2014, p. 32).

Actualmente las rutas de transporte que prestan servicio en Tecámac cuentan con 128 destinos, destacando el Distrito Federal como destino final con el 59\% de las rutas; le sigue el municipio de Ecatepec con un 16\%, Naucalpan con el $8 \%$ y el $14 \%$ son rutas locales. Los recorridos de las rutas que tienen origen en el municipio de Tecámac y como destino el Distrito Federal realizan un recorrido promedio de $38 \mathrm{~km}$ y una duración del viaje en promedio de una hora con cuarenta minutos (Morán, 2014, p. 44).

El transporte en el municipio cuenta con una amplia variedad de rutas interurbanas, pero con una escasa cantidad de rutas que logren la conectividad al interior del municipio, producto del incremento de la demanda de viajes de los residentes de los conjuntos habitacionales. Sólo en cuatro de los nuevos conjuntos habitacionales (Sierra Hermosa, Real del Cid, Valle San Pedro y Los Héroes Tecámac) existen empresas que proporcionan el servicio de transporte colectivo (Morán, 2014, p. 50).

Cabe aclarar que en México no se producen estadísticas de movilidad de manera regular. La Encuesta Origen Destino 2007 es la más reciente que se ha realizado para la ZMVV, sin embargo, una visión más actualizada de los hábitos de movilidad se complementa con otros estudios de caso realizados en el municipio.

\section{LOS HALLAZGOS DEL ESTUDIO DE CASO EN EL MUNICIPIO DE TECAMAC}

Se mencionó que una de las fuentes de información más importantes de esta investigación fue la Encuesta "Movilidad Residencial y tendencias electorales en Tecámac y Zumpango, Estado de México" (CIECAS, 2013) dirigida a jefes de familia residentes de los conjuntos habitacionales en ambos municipios, con un muestreo estratificado por asignación proporcional en los conjuntos habitacionales identificados en cada municipio. Lo que arroja 426 cuestionarios en Tecámac y 419 en Zumpango. Durante el levantamiento de la Encuesta (CIECAS, 2013), se pudo documentar las características del entorno urbano y el espacio de vida con algunos relatos de los entrevistados.

En Tecámac se entrevistaron 255 mujeres y 171 hombres, lo que revela una mayor disposición femenina para responder el cuestionario, además de que encontramos más mujeres en el hogar y en espacios públicos. El $65.7 \%$ de los entrevistados tenía entre de 25 y 44 años de edad. La juventud relativa está asociada al ciclo de vida familiar, ya que generalmente salen de otras áreas de la ciudad familias jóvenes en busca de vivienda, ante la saturación urbana y el alto costo de la vivienda principalmente en las delegaciones centrales del Distrito Federal. 
El 40.5\% los residentes de las unidades habitacionales, según la Encuesta, provienen del Distrito Federal y el 58.8\% del Estado de México. Sólo 8.3\% de los residentes son originarios del propio municipio. Ahora bien, de los residentes provenientes del Estado de México, el 27\% proviene del municipio de Ecatepec (municipio limítrofe); le sigue la Delegación Gustavo A. Madero (14.7\%), Nezahualcóyotl (6.9\%), la Delegación Cuauhtémoc (4.3\%) e Iztapalapa (4\%).

Estos hallazgos coinciden con otro estudio relacionado con los conjuntos habitacionales Héroes Tecámac y Sierra Hermosa, estimando que el 76 \% de la población se traslada diariamente a las delegaciones centrales del Distrito Federal así como a municipio de Ecatepec en el Estado de México y Tizayuca en el Estado de Hidalgo tanto para sus compras como para sus actividades de esparcimiento, pues señalan que dentro del municipio el acceso a dichas actividades es más costoso (García y Castañeda, 2013).

Los espacios periurbanos, se caracterizan generalmente por el predominio de actividades de escaso valor agregado y ligadas al pequeño comercio local; no existe una economía de aglomeración, ni aprovechan las ventajas de localización que indudablemente tiene el municipio. La información del Censo Económico (INEGI, 2009) lo corrobora, ya que el 64.6\% de las 8659 unidades económicas registradas, se dedican al comercio de pequeña escala y el 11.3\% a la manufactura. La composición del empleo es similar: de 27207 empleos, el 52\% se dedica al comercio al por menor, el 18.5\% a la manufactura, el $4.8 \%$ al comercio al por mayor y $4.2 \%$ a los servicios educativos.

Por lo tanto, la eficiencia que genera este esquema de urbanización disperso es muy escasa, porque una gran parte de la actividad local se desarrolla a partir del lugar de residencia. Quedó claro que los grandes desplazamientos se realizan por motivo de trabajo, lo que pone en evidencia que las actividades que mayor empleo generan no se localizan en el entorno local.

Así, más que una elección, la localización de la vivienda está determinada por la accesibilidad, no sólo entendida como la capacidad económica y el nivel de financiamiento que las familias puedan adquirir con base en su salario y su estabilidad laboral, sino por la localización de los conjuntos residenciales que se dirigen a los municipios que cuentan con disponibilidad de suelo urbanizable a bajo costo.

Respecto al lugar donde laboran, el 52.7\% de las 205 personas que aseguraron tener empleo, se desplazan diariamente al Distrito Federal, el 24.4\% refirió el propio municipio de Tecámac, 17.8\% otro municipio y el 5\% otra entidad. Así, los residentes de Tecámac, que laboran en el Distrito Federal se desplazan diariamente largas distancias y emplean más tiempo.

A partir de la Encuesta (CIECAS, 2013) también buscamos patrones de consumo cultural, entre los que destacan, las actividades recreativas. De los 422 entrevistados, 131 (31\%) satisfacen este tipo 
de necesidades en el propio conjunto residencial, refiriéndose principalmente a los parques públicos y los cines o ciertas actividades que ofrecen los centros comerciales cercanos; 41 personas aludieron que se dirigen a otro municipio, destacando nuevamente Ecatepec por su colindancia y por el mayor grado de concentración de actividades económicas, entre las que se encuentran cines, casas de cultura y otras. No obstante, el 15\% de los residentes de Tecámac se trasladan al Distrito Federal, lo cual nuevamente denota que su espacio de vida está fuera del entorno local.

Comparando los dos patrones de desplazamiento, por motivo laboral y para la realización de actividades de ocio, tenemos una mayor propensión a los desplazamientos de larga distancia (al Distrito Federal) por motivos laborales con respecto a los desplazamientos generados a partir de actividades culturales.

Suárez Lastra y Delgado (2010) realizan un estudio sobre el comportamiento de los desplazamientos residenciales en función del ingreso de los hogares en la ZMVM. Encuentran que los movimientos originados en cualquiera de los anillos de conurbación y que terminan en la ciudad central o el primer anillo, tienen medias de ingresos mayores a las de quienes no cambiaron de residencia en los anillos de origen y destino. Al contrario, las familias que provienen del cuarto y, en algunos casos, del tercer anillo, muestran ingresos menores que los de la población que mantuvo su lugar de residencia anterior.

La ciudad central recibió a los hogares con mayor ingreso. Los hogares que salieron de la ciudad central fueron, también, los de mayor ingreso respecto a la zona metropolitana, pero de menor ingreso respecto a quienes se mudaron ahí. Así, a medida que aumenta la distancia al centro, disminuye el ingreso medio de los cambios que se originan y tienen destino en cada contorno [...] los cambios que se originan en cada contorno representan un ingreso menor al de los cambios que tienen como destino esos mismos contornos [...] ello sugiere un patrón de expulsión de hogares de bajos ingresos hacia la periferia, generado por procesos de aburguesamiento (gentrification) en el lugar de origen (Suárez Lastra y Delgado, 2010, p. 780-781).

Analizando el comportamiento de la movilidad de la población por motivos residenciales en Tecámac, se puede plantear que la movilidad residencial no representa cambio en la demanda de bienes y servicios culturales. Por su origen socioeconómico y las pautas de consumo cultural, la población se adapta a lo que ofrece el entorno local de destino, toda vez que provee una oferta cultural similar a la que prevalecía en sus lugares de origen, ya que muy pocos provienen de las delegaciones centrales en las que se localiza una oferta cultural más especializada. 
Lo anterior tiene otra implicación importante en la movilidad cotidiana: los nuevos residentes no están dispuestos a desplazarse largas distancias para las actividades de ocio y recreativas, las cuales se realizan fundamentalmente en el entorno local.

Según el citado trabajo de García y Castañeda (2013, p. 617) el 48 \% de la población tarda más de una hora en trasladarse a su lugar de trabajo, el $48 \%$ utilizan de dos a tres transportes y el $20 \%$ utiliza auto particular, asimismo, el 60\% de la población gasta más de un salario mínimo en transporte, cuando el 64 tiene una nivel de ingresos entre uno y tres salarios mínimos. Ello nos da cuenta de una problemática generalizada en los tiempos y costos de la movilidad de los pobladores de esos conjuntos, lo cual impacta, sin duda, en sus tiempos de recreación y en sus prácticas culturales ya sean estas en un espacio específico, en medios de comunicación masiva o a través de las nuevas Tecnologías de la Información y la Comunicación (TIC). Es preciso considerar este escenario en cualquier acción gubernamental o social que pretenda incidir en la construcción de políticas culturales y educativas adecuadas a la realidad de estos conglomerados urbanos de la periferia de la metrópoli.

Esto también influye en el tiempo que los residentes están dispuestos a emplear para ir a su centro de trabajo, es un tiempo irrenunciable; en cambio, el tiempo destinado a actividades culturales o recreativas, es un tiempo prescindible. Siendo el tiempo un bien escaso, se puede afirmar que el tiempo que se sacrifica finalmente para otros destinos cotidianos como al trabajo o a la escuela, es el dedicado al descanso y a las actividades culturales.

Por motivos de estudio, existe un patrón de desplazamiento muy distinto: de las 166 personas que se desplazan por este motivo, $70 \%$ lo hacen dentro del municipio, porque se trata principalmente a llevar a los menores a las escuelas de educación básica, mientras que el 15.3\% se desplaza al Distrito Federal, preponderantemente a escuelas de educación superior y posgrado. El 12\% se dirige a otro municipio del Estado de México, sobresaliendo Texcoco, que concentra importante equipamiento educativo a nivel superior y posgrado. La localización de la escuela en un espacio próximo constituye un elemento importante de los espacios de vida.

Otro indicador del espacio de vida es el lugar donde realizan las compras cotidianas para el abasto familiar. De las 212 personas que realizan las compras cotidianas, para el abasto del hogar, 180 (85\%) aludieron al propio municipio, 17 en otro municipio, destacando Ecatepec; 13 en el Distrito Federal, personas que realizan sus actividades generalmente cerca del lugar donde laboran.

El análisis precedente sugiere que accesibilidad a los servicios culturales no sólo es una cuestión de la oferta cultural cercana, sino también, de tiempos y recursos. La fragmentación urbana característica de Tecámac se expresa en dos dimensiones: fragmentación del espacio y fragmentación del tiempo, en el sentido de una reducción del escaso o nulo tiempo social del que disponen las 
personas para realizar actividades culturales o recreativas $y$, por tanto, para tener acceso a bienes y servicios culturales diversos y de calidad.

Cabe señalar que el costo del transporte en el Estado de México supera en promedio, el 75\% al costo del transporte en el Distrito Federal, de tal manera que la población más pobre, que enfrenta los rezagos sociales más severos, está condenada a la inmovilidad e impedida de ejercer su derecho a la ciudad, por no ser posible asimilar con su precario ingreso, los altos costos del transporte.

En seguida se analiza el grado en el que el municipio genera condiciones de accesibilidad en función del equipamiento cultural y su distribución espacial.

\section{EL EQUIPAMIENTO CULTURAL EN TECAMAC}

Según el Sistema de Información Cultural del Consejo Nacional Para la Cultura y las Artes, el municipio de Tecámac cuenta con 8 bibliotecas de las cuales sólo una se ubica en uno de los grandes conjuntos urbanos llamado Fraccionamiento Héroes de Tecámac. Cuenta con un Centro Regional de Cultura en el centro del municipio, 3 Universidades, dos públicas y una privada, un patrimonio ferrocarrilero (la estación Xolox, sin uso cultural específico) y tres complejos cinematográficos (Conaculta, 2015); complejos de las compañías dominantes del mercado de exhibición cinematográfica nacional, uno de Cinepolis y dos de Cinemex, con aproximadamente 20 salas de exhibición, cuya cartelera es similar a las de cualquier otros multiplex de la ciudad. Es decir, con el dominio de la oferta hollywoodense, con lo que ello implica en la oferta cultural del municipio y de quienes habitan los nuevos conjuntos habitacionales (Mapa 2).

Estos datos se complementan con otros expuestos en el Plan de Desarrollo Municipal (PDM, 2007), en el cual se establece que prácticamente en todos los aspectos relacionado con el equipamiento de cultura, deporte y entretenimiento (excepto las bibliotecas), el municipio tiene un déficit considerable en términos de espacio construidos en todo los demás aspectos. El único criterio definido: metros cuadrados por habitante es absolutamente insuficiente para revelar la problemática del equipamiento y la gestión cultural en el municipio, especialmente en los conjuntos habitacionales.

De tal manera que no existen indicadores adecuados para ponderar el grado de acceso a la cultura; tampoco los objetivos y la estrategia cultural son claros, no existe un proyecto integral con acciones y políticas municipales con la visión de promover la cohesión social, identidad local, el uso cultural de los espacios públicos, que procure una oferta cultural diversa y entornos educadores orientados a mejorar la habitabilidad de los distintas localidades del municipio. 
Mapa 2. Equipamiento cultural en Tecámac y Ecatepec.

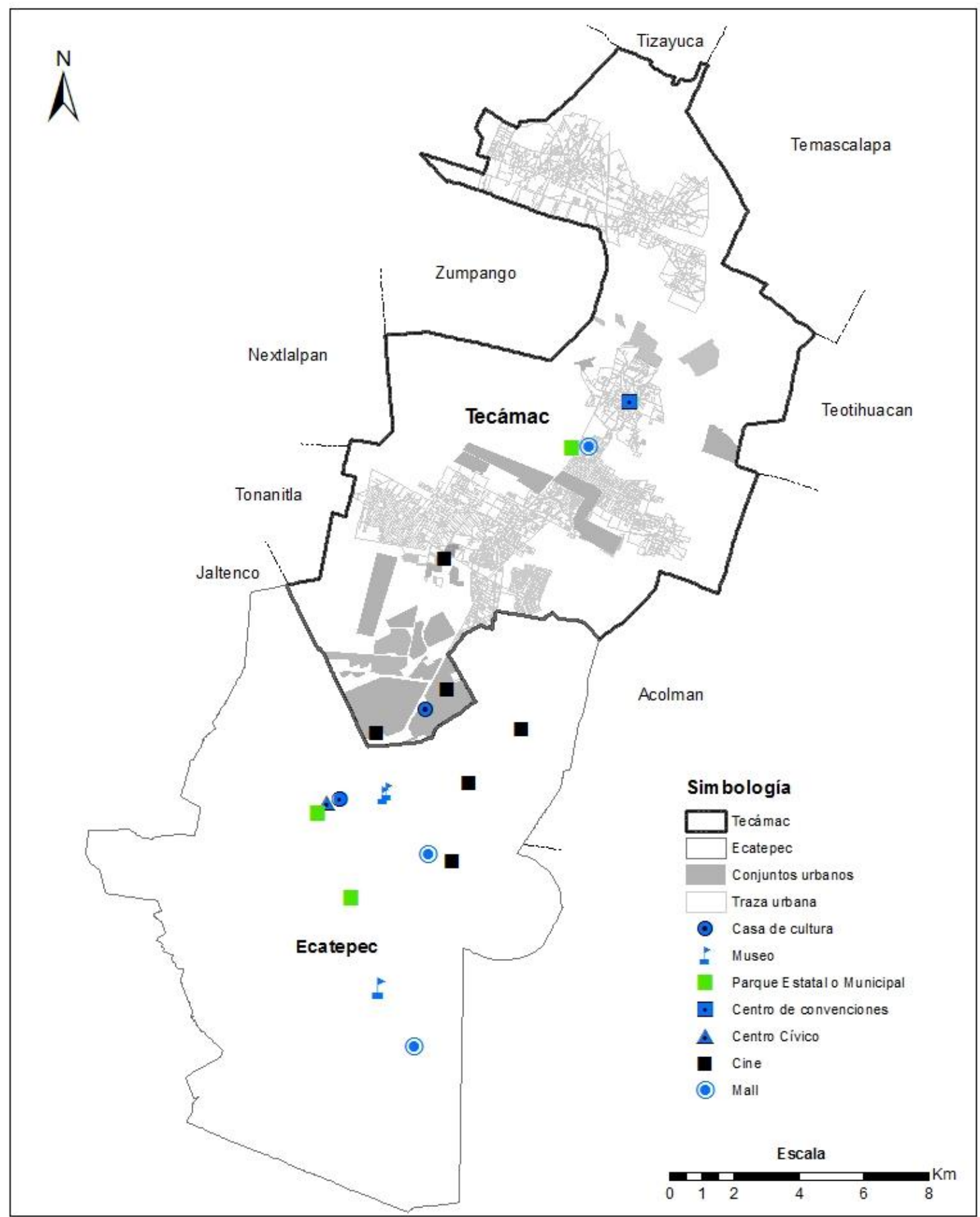

Elaboró: Mtro Marco A Morán V

Fuente: INEGI, Marco Geoestadístico Estatal, 2010

INEGI, Marco Geoestadístico Municipal, 2010

Fuente: Elaboró Marco Morán V. con base en: INEGI, Marco Geoestadístico Estatal y Municipal 2010. 
El Mapa 2 pone en evidencia dos aspectos en torno al acceso a la cultura. En primer lugar la muy escasa dotación y dispersión de servicios culturales; en segundo lugar, la fragmentación urbana se manifiesta en la nula dotación de servicios culturales y la escasa accesibilidad hacia el resto del municipio que tienen las áreas centro y norte del municipio; por su parte, la región sur, muestra un grado relativamente mayor de accesibilidad a cines y casas de cultura, lo que sugiere una relación funcional más estrecha con el municipio de Ecatepec.

Este aspecto revela la falta de regulación de las empresas inmobiliarias y la escasa capacidad de gestión de los gobiernos municipales, se limitan a dotar del equipamiento mínimo y restan importancia al equipamiento deportivo, de entretenimiento y cultural, a pesar de su importante repercusión espacial y funcional. En este caso los equipamientos de proximidad y los de centralidad son relativamente escasos en función de las características sociodemográficas del municipio de Tecámac.

Los equipamientos de centralidad son edificios únicos con dimensión de capitalidad y no de territorialidad, destinados a atraer a todas las personas interesadas y no sólo a los vecinos del entorno próximo, normalmente con una arquitectura singular y de gran tamaño, unifuncionales, centrados en los productos y no en los procesos, y de carácter específicamente cultural. En su mayoría se ubican en los cascos viejos de las ciudades o en espacios privilegiados de la zona urbana. Pueden ser un teatro, un museo, una gran biblioteca, un auditorio, un centro cultural, etc. (López de Aguilera, 2000, p. 238239). Tal es el caso del Auditorio Municipal y de la Casa de Cultura de la cabecera municipal de Tecámac.

Los equipamientos de proximidad tienen un carácter local, territorial, de servicios básicos para la acción cultural; normalmente se trata de locales polivalentes y socioculturales, dirigidos al consumo local centrado en los procesos que se generan más que en la calidad de los productos. De tal manera que los equipamientos de proximidad constituyen un elemento importante en la creación de un entorno habitable pues en ellos no sólo se ocuparían para las actividades culturales sino podrían ser espacios polifuncionales, que albergaran diversos servicios (educativos, sociales, recreativos, gestión ciudadana, etc.) y se convirtieran en referentes o articuladores de ciertas actividades en el interior de los conglomerados habitacionales. Tales son los casos de la explanada de la sección jardines del Conjunto Habitacional Los Héroes Tecámac, o el Centro de Convenciones y el gimnasio municipal ubicados en la cabecera municipal.

Al insuficiente equipamiento urbano se suma el limitado uso del espacio público, elemento necesario para favorecer la vida pública y los lazos comunitarios. En el caso de Tecámac, el espacio público no es valorado como ámbito para la promoción y difusión cultural. El problema no sólo es la 
falta de acciones en ese espacio, o el limitado número de ellas, también tiene que ver con la privatización por parte de micropoderes fácticos, que paulatinamente se han apropiado de ellos, tanto en los nuevos conjuntos urbanos como en el espacio público de los pueblos y colonias del municipio.

Por su parte, los centros comerciales se han convertido en una de las opciones de mayor preponderancia para "estar en la ciudad" sin necesidad de recorrer largas distancias metropolitanas, en un entorno controlado por cámaras y vigilantes, y con una oferta comercial y de servicios similar a la de otras lugares de la ciudad, del país, del mundo, mediada esta imagen global por las condiciones económicas de la zona. Las personas se introducen a una "una ciudad encapsulada", cuyas prácticas sociales y culturales modificaran paulatinamente su relación con su entorno y la ciudad. En este sentido Beatriz Sarlo expone que "el miedo de la ciudad y el miedo en la ciudad, el éxodo a barrios cerrados, a enclaves que simula aldeas o suburbios bajo control, el abandono de los espacios abiertos a causa de sus acechanzas" son resultado de una ciudad fuera de control. Agrega que "a esta forma de enfrentar un conjunto de cambios, que sucedieron en todas las ciudades del mundo, el mercado ofreció su creación: el shopping, un espacio público de gestión privada" (Sarlo, 2009, p. 23).

El centro comercial es un elemento urbanístico que embona con precisión a los conjuntos urbanos como los ubicados en la zona de Tecámac. Emilio Duhau (2008) lo plantea así:

Dadas las grandes dimensiones de mucho de estos conjuntos, constituyen grandes concentraciones de población que requieren de equipamientos y conforman una demanda concentrada de bienes y servicios. De allí, que los desarrollos vayan acompañados de la construcción de centros comerciales [...] y la total sustitución del sector público en la provisión de infraestructuras y equipamientos... (Duhau, 2008, p. 23).

Constatamos que los bienes culturales a los que accede la población, en general, son a través de los medios masivos de información o por medio de las nuevas tecnologías de la información y la comunicación (TIC). En otros espacios de la ciudad la cultura situada es más accesible a los habitantes en la dimensión local, en los nuevos espacios habitacionales esto es limitado, no sólo por la falta de equipamientos y espacios en dónde acudir sino a la muy limitada oferta cultural que existen en su interior como en el entorno inmediato. Aunado a ello, los pocos espacios orientados a la producción, difusión, consumo de bienes y servicios culturales y a la educación artística padecen la deficiente gestión gubernamental.

Llama la atención, que en algunos relatos, los residentes refieren como actividades lúdicas caminar o hacer ejercicio en el parque, ir al cine o ir a tomar un café. Para ir al cine se desplazan a los centros comerciales que se ubican dentro del municipio o en Ecatepec. Se observó que en los centros comerciales, no obstante la limitada oferta y calidad, existen actividades y espacios de carácter recreativo y en menor medida cultural. 
También es significativo el desplazamiento al Distrito Federal, para quien busca actividades más especializadas que Tecámac no ofrece, como cine de arte, conciertos, obras de teatro, exposiciones diversas, librerías, formación artística, entre otras, generalmente cercanas al lugar de trabajo.

\section{EL ACCESO A LOS BIENES Y SERVICIOS CULTURALES: ENTRE EL OLVIDO Y LA CASA}

Se han discutido ya los diferentes significados y sentidos de la movilidad y la vida cotidiana, de acuerdo con la realidad de las personas y la posibilidad de relacionarse e integrarse con el conjunto de la ciudad. Desde esta perspectiva se considera:

La movilidad como aquello que va a permitir a los individuos el acceso a la ciudad, a sus territorios, a las oportunidades de toda índole que ésta ofrece y, por supuesto, a los vínculos sociales tan importantes para los colectivos urbanos. Lo anterior nos conduce a una reflexión más profunda sobre el nexo, a veces oculto, entre la movilidad y el funcionamiento contemporáneo de la sociedad, entre quienes pueden desplazarse sin problema alguno y los que no pueden hacerlo. Trabajar, consumir o relacionarse con los otros implica necesariamente desplazarse. Entonces la inserción social de un individuo estará determinada en buena medida por la capacidad y las posibilidades que tenga éste para desplazarse (Le Breton, 2006, citado por Avellaneda y Lazo, 2011, p.49).

En la metrópoli contemporánea se ha pasado a un modelo de vida donde las diferentes actividades se hallan dispersas a lo largo de una extensa trama urbana, de tal suerte que la vida cotidiana no se organiza en el entorno local (espacio de proximidad) sino a lo largo y ancho del espacio metropolitano, por lo que "...en la ciudad contemporánea el espacio cotidiano se vuelve mucho más difuso y disperso" (Avellaneda y Lazo, 2011, p. 55). A la luz de tal reflexión, es posible vincular los elementos de la estructura urbana, en especial el equipamiento cultural y recreativo, que favorece la convivencia y la cohesión social.

El modelo de urbanización descrito en la ZMVM ha ignorado la perspectiva multifuncional del espacio y una serie de elementos de los que debe estar dotado el entorno local para construir los espacios de vida y los referentes culturales que otorgan identidad a los grupos sociales; en este caso se encuentra el Municipio de Tecámac, que, en uso de las facultades de un gobierno local no tiene la capacidad técnica y financiera ni el equipamiento adecuado y suficiente para atender la demanda de bienes y servicios urbanos, en especial, los culturales. Ello propicia que los nuevos pobladores, residentes de los conjuntos habitacionales, en su proceso de adaptación tampoco demanden nuevos servicios culturales. 
En relación con el ciclo de vida familiar, cambia la precepción conforme se desarrollan las familias en el sentido de la edad y expectativas por rango de edad., lo que se vincula con las necesidades y demanda de carácter cultural y recreativas. La oferta cultural debe corresponder a las características demográficas y socioculturales de los grupos de población. Debe procurar diversidad. Sin embargo es preciso anotar la necesidad de que en el espacio local se generen las condiciones para atender las expectativas en un marco de diversidad cultural.

A partir de estas reflexiones, podemos afirmar que en Tecámac, y en diversos municipios de la periferia lejana de la metrópoli no se puede hablar de una política cultural municipal definida. Los que se observa son planteamientos desarticulados, con acciones fragmentadas y sin idea clara de los ejes centrales para atender las necesidades de los habitantes de un municipio con una realidad diversa, multicultural, territorialmente fragmentada y diferenciada socioculturalmente (nuevos fraccionamiento, pueblos y colonias populares). Una revisión del Plan de Desarrollo Municipal 20132015 y del Bando Municipal 2015 de Tecámac, revela la inexistencia de planteamientos que coloquen a la cultura como un tema de interés gubernamental, salvo las menciones formales, que en términos reales no pretenden atender las necesidades culturales y de recreación de las diversas poblaciones del municipio ni se observa acciones que busquen potenciar las capacidades de comunidades culturales y artísticas con propuestas tradicionales o innovadoras. En consecuencia, la oferta cultural y artística de los espacios culturales del municipio es limitada y reducida en los cánones tradicionales de este tipo de instalaciones a nivel nacional.

Una visita a la Casa de la Cultura permitió identificar con precisión la oferta de actividades que promueve: defensa personal, baile regional, clases de canto, estilismo, talleres de teatro y certificaciones del Instituto Nacional para la Educación de los Adultos (INEA). Las instalaciones cuentan con un espacio ex profeso para obras de teatro que está subutilizado, ya que el personal mencionó que las sólo se habían presentado dos obras de teatro "Vaselina" y "Blanca nieves y los siete enanos" hacía más de un año. En algunos de ellos existen formalmente clases de ballet folclórico y clásico, de canto y de teatro; sin embargo, estas actividades son irregulares en su impartición y consideradas como un pasatiempo.

Un residente de Los Héroes relata:

Debido a la falta de oferta de eventos culturales de mayor contenido, he optado por ir al municipio vecino de Ecatepec dónde se ofrecen eventos con más regularidad en su centro cívico o a la ciudad de México a la Cineteca Nacional o a los diferentes teatros.

La investigación de campo revela que los principales lugares para actividades lúdicas y de esparcimiento a los que acuden los habitantes de Tecámac se localizan en plazas comerciales, parques, salas culturales, museos y zonas arqueológicas localizados en los municipios de Ecatepec, 
Coacalco, Teotihuacán y la ciudad de México, y en menor medida en el propio municipio de Tecámac. Ello muestra que el equipamiento cultural es insuficiente, inadecuado, mal equipado, el funcionamiento y calidad de los servicios prestados es variable en cada espacio, y en muchos casos las instalaciones son subutilizadas.

Más allá del equipamiento, la situación revela la inexistencia de una política y gestión cultural adecuada que atienda las necesidades de los grupos sociales que habitan el municipio. A luz de la Encuesta y el trabajo de campo, se puede demostrar que el entorno local no facilita ni promueve la cohesión social en torno a la cultura. Poner en marcha acciones de política cultural sin tener soportes estables dificulta la continuidad y estabilidad de una política.

Otro elemento a considerar es el escaso vínculo social que de los nuevos pobladores y su relación con el territorio circundante, el espacio social al cual llegan en condición de avecindados o fuereños. El generar mecanismos de integración al lugar donde se llega y la convivencia interna y externa con los otros son aspectos que requiere de una intervención en diversas dimensiones. Los espacios públicos, la acción sociocultural y los equipamientos urbanos de proximidad pueden contribuir a mejorar la sociabilidad de los habitantes y su calidad de vida, la cual es afectada por un ambiente circundante agreste, un espacio público conflictivo en el interior de los conjuntos urbanos y una vivienda con espacios reducidos a la "vivienda mínima".

Los pocos espacios públicos y equipamientos culturales en funcionamiento de estas unidades tienen limitada capacidad de ofrecer actividades diversas orientadas al arte y la cultura o a otras actividades vinculadas con el esparcimiento de los diferentes sectores de la población. La exigua oferta cultural y las pocas instalaciones para esas actividades se encuentran principalmente en el centro del municipio, en el pueblo de Tecámac lo cual obstaculiza aún más el acceso a estas actividades pues por la organización y los tiempos de la vida cotidiana de sus habitantes les hace casi imposible asistir o permanecer en ellas tiempo considerable. Los contados equipamientos de centralidad como el auditorio municipal o el Centro Regional de Cultura, por sus características, tendría poca capacidad para generar procesos culturales (producción, difusión y consumo) en las decenas de miles de familias que pueblan el municipio. Actualmente son espacios, el primero para actividades masivas, festivales, entre otras; o el segundo, para actividades culturales de públicos reducidos.

Otro relato de un residente de Tecámac señala: "Los residentes del municipio interesados en la cultura en ocasiones se trasladan a otros lugares de la ciudad, en los que sí existe una oferta regular, diversa y aunque a precios diferenciados. Para ello recorren largas distancias, incorporando a su experiencia cultural o artística, el costo de la distancia, el tiempo y la inseguridad que significa los recorridos entre su unidad habitacional y el Distrito Federal. 
En el trabajo de campo se observó la gran centralidad que ejerce el municipio contiguo de Ecatepec, el municipio más poblado de México. Los fines de semana las plazas comerciales de Ecatepec están saturadas: Plaza Aragón, Plaza las Américas, Plaza Center, Plaza Sendero. También se observó gran concentración de familias en los parques públicos: Parque Ecológico Ehécatl y Parque Siervo de la Nación, los cuales cuentan con gran cantidad de actividades. Existe también una oferta de museos como: el Museo de Historia Natural y el Museo Puente del Arte; finalmente encontramos en este municipio contiguo a Tecámac casas de cultura con una amplia oferta de actividades, como lo es el Centro Regional Cultural José Ma. Morelos y Pavón o visitas guiadas en el centro comunitario casa Morelos, finalmente el centro cívico de Ecatepec tiene una amplia actividad en lo que respecta a eventos.

En Coacalco, otro municipio cercano a la zona de estudio, también se localizan plazas comerciales que han ampliado su oferta de esparcimiento, al implementar juegos mecánicos y pistas de hielo. Finalmente otro municipio donde se trasladan las personas para actividades lúdicas y culturales es el municipio de Teotihuacán donde se localizan la zona arqueológica.

Los habitantes de los conjuntos estudiados que asisten a actividades recreativas en el Distrito Federal representan el (14.7\%) según la Encuesta (CIECAS, 2013). En cambio, aproximadamente un 30 $\%$ asiste a espacios dentro del municipio de Tecámac y otro 30\% realiza esas actividades en el propio desarrollo.

La movilidad implica entonces los procesos de construcción o apropiación del territorio y su uso diferencial, porque expresa la dimensión espacial de los procesos sociales y las prácticas cotidianas que permiten el acceso a los lugares de trabajo, de educación, de atención de la salud, visitas sociales, compras, ocio y recreación (Blanco, Bosoer y Apaolaza, 2014, p. 2).

\section{REFLEXIONES FINALES}

El análisis presentado pone sobre la mesa valiosos hallazgos. Inicialmente se esperaba que los nuevos residentes fueran portadores de pautas de consumo cultural distintas a las que prevalecían en el entorno local. El estudio muestra que si bien existe un proceso de diferenciación social, ésta sucede entre los nuevos residentes y los pobladores originarios, ya que se trata de productores rurales subsumidos en una situación de pobreza y aislamiento social.

La fragmentación urbana propiciada por el modelo urbanístico prevaleciente y por la política de vivienda, se traduce en la escasa interacción de los residentes de los conjuntos habitacionales respecto a los pobladores de las localidades tradicionales. 
Así, la movilidad residencial no significa un cambio en las pautas de consumo cultural de los nuevos residentes, porque siguen disfrutando del cine ubicado en el centro comercial, o de las pocas actividades que ofrecen las casas de cultura. La explicación que subyace en tal comportamiento es que el perfil socioeconómico de la población que finalmente tuvo acceso a la vivienda social en Tecámac, proviene de áreas de la ciudad que ofrece patrones de consumo cultural similares, promovidos desde las instituciones en el marco de una política cultural dominante, que ignora la diversidad cultural y la importancia de la cultura desde el ámbito local.

Las acciones o la inacción de las políticas urbanas del gobierno federal, estatal y municipal han generado un entramado social y urbano conflictivo. Lejos de integrar a la población pobre de la periferias a los recursos que ofrece el sistema urbano, la política habitacional seguida en las dos décadas anteriores ha reforzado la segregación y ha potenciado la formación de una cultura de la segregación que se expresa a través de la descomposición del tejido social (surgimiento de bandas, altos índice de desempleo, pobreza, cooptación de la comunidad por las redes del narcotráfico, etc.).

No se puede hablar de una política cultural municipal definida. Los que se observa son planteamientos desarticulados, con acciones fragmentadas y sin idea clara de los ejes centrales para atender las necesidades de los habitantes de un municipio con una realidad diversa, multicultural y territorialmente fragmentada y diferenciada socioculturalmente (nuevos fraccionamiento, pueblos y colonias populares). En lo que se ha obtenido de información (Plan de Desarrollo Municipal 2013-2015 y Bando Municipal 2015) no hay planteamientos que ubiquen a la cultura como un tema de interés gubernamental, salvo las menciones formales, que en términos reales no pretenden atender las necesidades culturales y de recreación de las diversas poblaciones del municipio ni se observa acciones que busquen potenciar las capacidades de comunidades culturales y artísticas con propuestas tradicionales o innovadoras.

Las expresiones de cultura popular, de las bellas artes o de vanguardia no son consideradas como parte fundamental de la oferta cultural oficial o dominante; asimismo las diversas expresiones artístico culturales producida o recreadas por la comunidad tampoco son materia relevante de intervención o gestión cultural. Hay una reproducción acrítica de prácticas, contenidos y valores de la cultura hegemónica, principalmente de la cultura de masas producidas o difundida por los medios de comunicación masiva y corporaciones del entretenimiento nacionales y extranjeras.

Estos agentes económico- culturales tienden a descalificar, degradar, caricaturizar y limitar la difusión de expresiones de la cultura popular de los grupos subalternos. Así pues los habitantes del municipio de Tecámac no cuentan con una oferta de actividades culturales diversa en modalidades, 
calidad y cantidad adecuada a los requerimientos de los diferentes grupos de la población que habitan actualmente el municipio.

La gestión de la cultura desde el ámbito local tiene que pasar por el reconocimiento de la diversidad, fomentar la cohesión social, valorar los intangibles de las comunidades y proporcionar elementos que transformen el entorno en un espacio más habitable. Para enfrentar la problemática de acceso a bienes y servicios culturales es importante considerar una política y gestión culturales acordes a las necesidades locales, en las que incorporé la dimensión del espacio público como un ámbito fundamental para articular las acciones de política pública y la satisfacción de las necesidades de los ciudadanos de estos nuevos entorno urbanos.

El espacio público debe fortalecer el encuentro de grupos sociales, diversas expresiones de la cultura, la construcción de lazos de confianza y de proximidad y de identidad; finalmente de territorio

Finalmente el problemas de las políticas culturales de base territorial no es solamente de oferta o equipamientos, es estructural, de gestión y de concepción: qué finalidad tiene la cultura, qué tipo de culturas impulsar y difundir, cómo gestionarlas y qué públicos y tipos de habitantes se debe atender a partir de sus necesidades y condiciones materiales de existencia. Además de incorporar a esta temática el vínculo necesario entre políticas culturales, educativas y de bienestar social, perspectiva que está lejos de estar presente en el caso que nos ocupa.

\section{REFERENCIAS}

ALGUACIL, Julio (2001) Calidad de vida y Modelo de Ciudad Boletín CF+S Biblioteca Ciudades para un Futuro más Sostenible num. 15 Calidad de vida urbana: variedad, cohesión y medio ambiente, Instituto Juan de Herrera. Madrid. Consulta electrónica (4 de mayo de 2014): http://habitat.aq.upm.es/boletin/n15/ajalg.html

AVELLANEDA, Pau y Lazo, Alejandra (2011) Aproximación a la movilidad cotidiana en la periferia pobre de dos ciudades latinoamericanas. Los casos de Lima y Santiago de Chile Revista Transporte y Territorio № 4, Universidad de Buenos Aires, pp. 47-58, Consulta electrónica (3 de abril de 215): www.rtt.filo.uba.ar/RTT00404047.pdf

BLANCO, J., Boosoer, L. y Apaolaza, R. (2014) Movilidad, apropiación y uso del territorio: una aproximación a partir del caso de Buenos Aires Scripta Nova, Revista Electrónica de Geografía y Ciencias Sociales, Universidad de Barcelona, vol. XVIII, núm. 493 (06), 1 de noviembre de 2014

BOILS, Guillermo (2008). Segregación y modelo habitacional en grandes conjuntos de vivienda en México, en Cordera, Rolando, Ramírez Patricia, Ziccardi, Alicia (coords.). Pobreza, desigualdad y exclusión social en la ciudad del siglo XXI. México: UNAM, Siglo XXI, pp. 273-287

CIECAS (2013) Encuesta Movilidad residencial, tendencias electorales y espacios de vida en Tecámac y Zumpango, estado de México, Centro de Investigaciones Económicas, Administrativas y Sociales, Instituto Politécnico Nacional. 
CONACULTA (2015). Sistema de Información Cultural. México: Consejo para la Cultura y las Artes. Consulta electrónica (16 de marzo de 2015): http://sic.conaculta.gob.mx/index.php?estado_id=15\&municipio_id=81\&table=biblioteca\&disciplina=

COURGEAU, Daniel (1988): Méthodes de mesure de la mobilité spatiale. Migrations internes, mobilité temporaire, navettes. Institut National d'Etudes Démographiques, Paris, Francia.

DELAUNAY, Daniel y Dureau, Françoise (2004): Componentes sociales de la movilidad residencial en Bogotá en: Revista Estudios Demográficos y Urbanos Vol. 19 №55, enero-abril de 2004, El Colegio de México.

DUHAU, Emilio (2008). Los nuevos productores del espacio habitable, en Ciudades, núm. 49, julioseptiembre de 2008, pp. 21-27

DUHAU, Emilio y GIGLIA Angela (2010). Las reglas del desorden: habitar la metrópoli. México: Siglo XXI.

GARCÍA CANCLINI, Néstor. (coord.) (1993). El consumo cultural en México. Consejo Nacional para la Cultura y las Artes (CNCA), México.

GARCÍA, Guadalupe (S/a). La expansión metropolitana bajo la nueva vivienda en Tecámac, Estado de México 2000-2010. En Revista GeoSur 2(1): 47-67

GARCÍA, Guadalupe y CASTAÑEDA, Hellen (2013) El capital inmobiliario como planificador del territorio en México. Caso de estudio: Tecámac, Estado de México, 2000-2008. En Sánchez Ma. Teresa, Gerardo Bocco y José María Casado (coords.), La política de ordenamiento territorial en México: de la teoría a la práctica, México, Instituto Nacional de Ecología y Cambio Climático. Consulta electrónica (15 de octubre de 2014). http://www2.inecc.gob.mx/publicaciones/libros/699/capital.pdf

GIGLIA, Angela (2012). El habitar y la cultura. Perspectivas teóricas y de investigación. Barcelona: Anthopos, Universidad Autónoma Metropolitana-Iztapalapa.

GOBIERNO DEL ESTADO DE MÉXICO, Plan de Desarrollo Municipal de Tecámac, 2007. Gobierno del Estado de México, Secretaría de Desarrollo Urbano, consulta electrónica: http://www.edomex.gob.mx/sedur/estadisticas/conjuntos-urbanos.

INEGI (2000): XII Censo General de Población y Vivienda

(2005) II Conteo de Población y Vivienda

(2010) Censo de Población y Vivienda

JIRÓN, Paola y MANSILLA, Pablo (2014). Las consecuencias del urbanismo fragmentador en la vida cotidiana de habitantes de la ciudad de Santiago de Chile. EURE, 40 (121), 5-28.

LANGE, Carlos (2011) Dimensiones culturales de la movilidad urbana, Revista INVI № 71, mayo 2011, vol. 26 , p. $87-106$.

LÓPEZ DE AGUILERA, I. (2000). Cultura y ciudad. Manual de política cultural municipal. Gijón, Esp. Trea,

MIRALLES, Carme (2011). Dinámicas metropolitanas y tiempos de la movilidad. La región metropolitana de Barcelona, como ejemplo en: Anales de Geografía, vol. 31, núm. 1 pp. 125-145, Universidad Complutense, Madrid, España 
MÓDENES, Juan Antonio (2004). Movilidad residencial, trabajo y vivienda en Europa, Publicación electrónica Revista Geocrítica Scipta Nova Universidad de Barcelona, ISSN: 1138-9788.

MORÁN, Marco Antonio (2014) Movilidad y transporte público en el municipio de Tecámac, Estado de México Tesis de la Maestría en Economía y Gestión Municipal, Centro de Investigaciones Económicas Administrativas y Sociales, Instituto Politécnico Nacional, México, diciembre de 2014.

PRECIAT, Eduardo (1997). Equipamiento y desequilibrio territorial en Eibenschutz $H_{\text {., }}$ Roberto (coord.). Bases para la planeación del desarrollo urbano en la ciudad de México, tomo II: estructura de la ciudad y su región. México, UAM-X./Miguel Angel Porrúa editor.

SANTOS y Ganges, Luis y De Las Rivas, Juan Luis (2008). Ciudades con atributos: conectividad, accesibilidad y movilidad Ciudades $N^{\circ} 11$, Revista del Instituto Universitario de Urbanística, Universidad de Valladolid, ISSN 1133-6579, p. 13-32.

SANTOS, Luis y De Las Rivas, Juan Luis (2008). Ciudades con atributos: conectividad, accesibilidad y movilidad Ciudades $N^{\circ} 11$, Revista del Instituto Universitario de Urbanística, Universidad de Valladolid, ISSN 1133-6579, p. 13-32.

SARLO, Beatríz (2009). La ciudad vista. Mercancías y cultura urbana. Buenos Aires: Siglo XXI.

SUSINO, Joaquín (2001). Movilidad residencial y movilidad cotidiana en áreas urbanas, en M. Castañer, J. Vicente y G. Boix (coordinadores.), Áreas urbanas y movilidad laboral en España, Universidad de Gerona, pp. 141-163.

TARCHÓPULOS, Doris y CEBALLOS, Olga (2003) Calidad de la vivienda dirigida a los sectores de bajos ingresos en Bogotá, Centro Editorial Javeriano, Pontificia Universidad Javeriana, Bogotá.

VELAZQUEZ, Osvaldo (2011). Habitabilidad en el parque habitacional de la AGEB 010-9 del fraccionamiento ojo de agua, Técamac Estado de México. En Tlatemoani. Revista Académica de Investigación, núm. 6, junio, pp.1-13.

WIRTH, Louis (1938). El urbanismo como modo de vida en The American Journal of Sociology, vol. 44, julio de 1938, tr. Víctor Sigal, en: Bifurcaciones, Revista de Estudios Culturales Urbanos N² 2, otoño de 2005, Buenos Aires. Disponible en: http://www.bifurcaciones.cl/002/reserva.htm

ZULAICA, Laura y CELEMIN, Juan Pablo (2008); Análisis territorial de las condiciones de habitabilidad en el periurbano de la ciudad de Mar del Plata Argentina, a partir de la construcción de un índice y de la aplicación de métodos de asociación espacial. Revista de Geografía Norte Grande N 41, p.129-146.

Trabalho enviado em 19 de julho de 2015.

Aceito em 10 de setembro de 2015. 